\title{
Ohtahara syndrome due to bilateral perisylvian polymicrogyria
}

\author{
J Wanigasinghe ${ }^{1}$, N I Gamaathige ${ }^{2}$, S Sumanasena ${ }^{1}$, M Weerasekera ${ }^{2}$
}

Sri Lanka Journal of Child Health, 2014; 43(1): 57-58

(Key words: Ohtahara syndrome; perisylvian polymicrogyria)

Neonatal epileptic encephalopathy is a rare cause of neonatal seizures. There are two main types. Early myoclonic epileptic encephalopathy presents with multifocal myoclonia as the predominant seizure type. Epileptic encephalopathy of early infancy or Ohtahara syndrome presents mainly with tonic seizures. Ohtahara syndrome commonly occurs secondary to underlying structural abnormalities in the brain. We report a case of Ohtahara syndrome due to bilateral perisylvian polymicrogyria (PMG).

\section{Case report}

A twenty day old baby boy was transferred with refractory convulsions since the second day of life. Following an uncomplicated pregnancy he was born at term by emergency caesarean section due to lack of progress. His birth weight was $3220 \mathrm{~g}$ and Apgar scores were normal. Parents were nonconsanguineous and there was no family history of neonatal convulsions.

He had two forms of seizures, brief focal convulsions followed by bilateral convulsive movements and tonic seizures with extension of both upper limbs. They occurred many times (15$35 /$ day) increasing in frequency in spite of multiple anticonvulsants. His EEG was grossly abnormal with bursts of moderate to high amplitude irregular spike waves followed by prolonged segments of background attenuation giving rise to typical burst suppression pattern. The inter-burst intervals ranged between 6-15 seconds.

He had normal system and neurological examination, good sucking reflex in between episodes with normal swallowing. No dysmorphism or neuro-cutaneous manifestations were noted. A moderately high C-reactive protein (CRP) of $48 \mathrm{mg} / 1$ (in spite of other negative septic markers) resulted in treatment as for neonatal meningitis with empirical antibiotics for a total of 3 weeks. Cerebrospinal fluid (CSF) examination was normal (normal full report, negative bacterial antigens, culture, and antigen for HSV). Blood investigations for sugar, serum electrolytes, renal functions, liver functions, and arterial blood gas were normal. Computed tomography (CT) of brain showed no abnormality. However, the MRI brain performed at two weeks of life showed presence of bilateral perisylvian polymicrogyria (Figure 1).
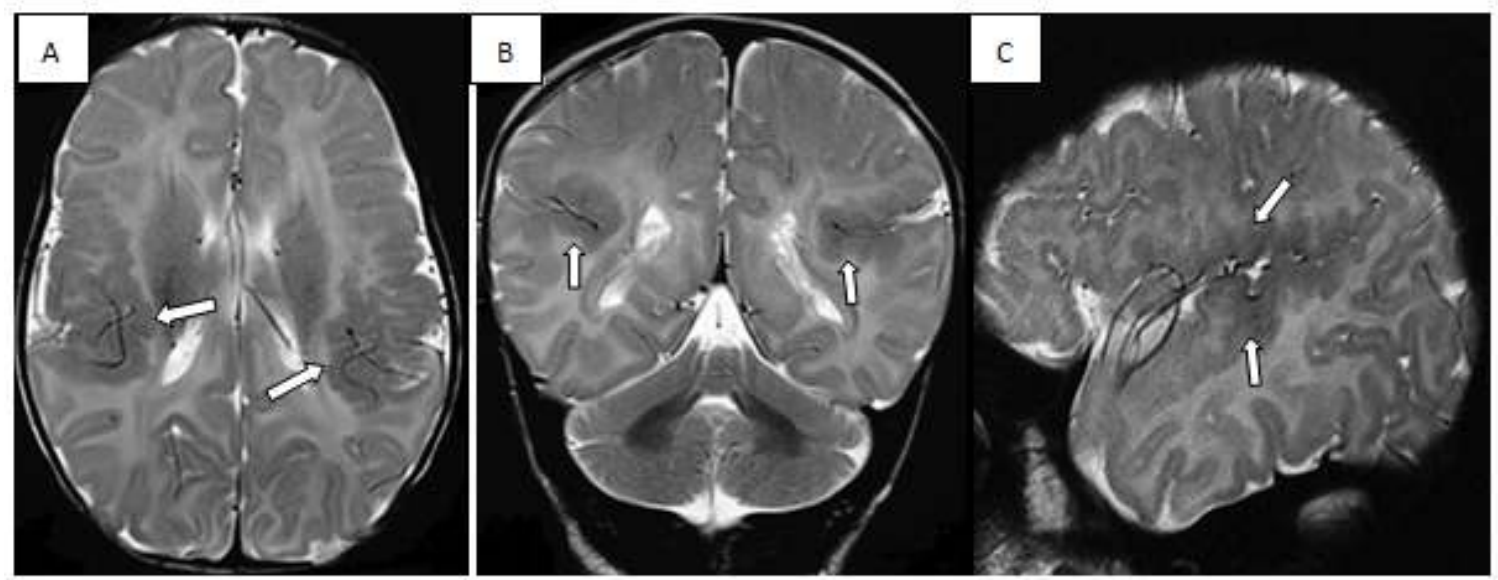

Figure 1: MRI appearance of bilateral perisylvian polymicrogyria showing increased thickness of grey matter giving rise to "cobblestone appearance" with irregular outline of inner and outer cortex. It manifests as decreased intensity on $T 1$ in $A$ - axial, $B$ - coronal and $C$-sagittal images of brain (white arrows)

\footnotetext{
${ }^{1}$ University of Colombo

${ }^{2}$ Sri Jayawardenapura General Hospital
}

(Received on 28 February 2013: Accepted after revision on 19 April 2013) 
This appeared as thickened cortex in the perisylvian regions (extending from the insular region to posterior margin of lateral sulcus) with numerous small closely packed gyri and shallow sulci, hypo intense on T1; hyper intense on $\mathrm{T} 2$ and FLAIR images. As congenital cytomegalovirus (CMV) infection is a recognised cause the CMV antibody status was assessed. Though they were markedly elevated in both mother $(>2500 \mathrm{AU} / \mathrm{ml})$ and the baby $(880 \mathrm{AU} / \mathrm{ml})$ at 2 months of age, repeat assay in the baby at 6 months $(80.15$ $\mathrm{AU} / \mathrm{ml}$ ) excluded this as possible underlying cause. $2 \mathrm{D}$ echo was normal and thymic shadow was seen on x-ray excluding Di George syndrome.

Seizures continued to occur without improvement despite multiple anticonvulsant therapy including intravenous phenobarbitone, phenytoin, midazolam infusions, topiramate and leveteracetam. Subsequently he developed West syndrome at three months of age which responded to hormonal therapy for a short duration. At 6 months of age he continued to have spasms and focal seizures daily, but at a lesser frequency. He had a prolonged focal status epilepticus during a febrile illness,

Development assessment at 6 months chronological age revealed a developmental age less than two months. Two episodes of severe bronchospasm requiring intensive care and prolonged hospital stay could have contributed to this severe delay in development. He showed no evidence of weakness of facial muscles, difficulty in swallowing or frequent drooling which is described as part of "perisylvian syndrome" in those diagnosed with bilateral perisylvian PMG in infancy.

\section{Discussion}

Polymicrogyria is a malformation of cortical development in which the process of normal cerebral cortical development is disturbed late in the stage of neuronal migration or early in the stage of cortical organization. The cortex on MRI appears thickened and bumpy due to multiple, abnormally formed gyri. The gyri show multiple small convolutions and too few sulci. This abnormal thickness of the cortex is due to fusion of the adjacent miniature gyri which have piled upon one another giving rise to a "cobblestone appearance" 1,2 . Histologically it is associated with derangement of the normal six-layered lamination of the cortex, an associated derangement of sulcation, and fusion of the molecular layer across sulci. Polymicrogyria affects variable portions of the cerebral cortex: it may be focal, multifocal, or diffuse; it may be unilateral, bilateral and asymmetrical; or bilateral and symmetrical. It is graded into four depending on the extent of cortical involvement (Grade 1 the most severe) ${ }^{2}$.
Polymicrogyria in $80 \%$ of cases occurs in the perisylvian region and is known sometimes as "perisylvian syndrome". Here the polymicrogyria is noted symmetrically in the perisylvian regions (Figure 1). The sylvian fissures are wide and often under developed. Sagittal images may show posterior extension of the sylvian fissure, exposure of the insula and apparent thickening of the cortex. The bodies of the lateral ventricles show inverted appearance, typical of this disorder. Other regions of involvement include frontal lobe $(70 \%)$, parietal $(63 \%)$ temporal (38\%) and occipital in $7 \%{ }^{3}$.

This is a polymorphic syndrome due to multiple aetiologies including congenital cytomegalovirus syndrome, Di George syndrome, Aicardi syndrome, in utero ischaemia and an array of specific gene mutations. A detailed family history is vital in the assessment of such patients.

Perisylvian syndrome may have a spectrum of clinical manifestations depending on the severity (extent of cortical involvement) and age at presentation. These include delayed milestones, epilepsy, variable intellectual disability and abnormal EEG. They may have other manifestations such as a developmental pseudobulbar palsy (oropharyngeal dysfunction and moderate to severe dysarthria) commonly seen in those presenting early ${ }^{4}$. Some have arthrogryposis and or lower motor neuron disease. In our patient it presented as an Ohtahara syndrome with predominant tonic seizures and typical EEG findings. Early presentation as well as bilateral involvement like in our patient is rare and is known to be associated with a poorer prognosis.

\section{References}

1. Barkovich AJ, Kuzniecky RI, Jackson GD, Guerrini R. A developmental and genetic classification for malformations of cortical development. Neurology 2005; 65:1873 -7. http://dx.doi.org/10.1212/01.wnl.0000183747. $05269.2 \mathrm{~d}$

2. Barkovich AJ. Current concepts of polymicrogyria. Neuroradiology 2010; 52:479 -87 .

http://dx.doi.org/10.1007/s00234-009-0644-2

3. Barkovich AJ, Hevner R, Guerrini R. Syndromes of bilateral symmetrical polymicrogyria. American Journal of Neuroradiology 1999; 20:1814 -21.

4. Taneja S, Chaturvedi AK. Bilateral Perisylvian Syndrome- A Case Report. Indian Journal of Radiology and Imaging 2006; 16(2):169-71. http://dx.doi.org/10.4103/0971-3026.29079 